\title{
Structural and Oxidation Properties of Plasma Lipoproteins from Different Phenotypes: FT-IR and ESR Study
}

\author{
Dubravka Krilov, ${ }^{\text {a,* }}$ Marin Kosović, ${ }^{\text {a Jasminka Brnjas-Kraljević, }}{ }^{\mathrm{a}}$ \\ Kristina Serec, ${ }^{\mathrm{a}}$ and Željka Vogrinc ${ }^{\mathrm{b}}$ \\ ${ }^{a}$ University of Zagreb School of Medicine, Šalata 3, 10000 Zagreb, Croatia \\ ${ }^{\mathrm{b}}$ University Hospital Centre Zagreb, Kišpatićeva 12, 10000 Zagreb, Croatia
}

RECEIVED FEBRUARY 1, 2013; REVISED JULY 2, 2013; ACCEPTED JULY 18, 2013

\begin{abstract}
Subclasses of lipoproteins VLDL, LDL and HDL were isolated from plasma of two different phenotypes, A and B. Phenotype B is present in people with diabetes, atherosclerosis and higher risk for cardiovascular disease. Lipoproteins were studied by FT-IR spectroscopy to investigate conformational and structural differences reflected in spectra. Differences were observed in spectra of LDL and VLDL. In protein domain differences were observed in amide I band profile and discussed in terms of conformational change of apo B-100 in small LDL and large VLDL particles. The changes in lipid bands from phenotype $\mathrm{B}$ are associated with differences in core composition and lipids' ordering in monolayer. The study of slow oxidation was done by measuring the oxygen consumption in lipoprotein solutions. Oxidation was faster in HDL and VLDL from phenotype B, while for LDL it was similar for A and B samples and significantly faster than in other two lipoproteins. (doi: 10.5562/cca2236)
\end{abstract}

Keywords: lipoprotein subclasses, phenotype, FT-IR spectroscopy

\section{INTRODUCTION}

Plasma lipoproteins had been extensively studied due to their role as transporters of hydrophobic compounds, lipids and sterols through plasma. All lipoproteins are supramolecular particles composed from hydrophobic core and lipid monolayer with one or more apolipoproteins on its surface. Several classes of lipoproteins are known which differ in size and composition of lipids and proteins. These structural properties are connected with their different metabolic roles. ${ }^{1}$ Three types of particles were studied here: very low density lipoprotein (VLDL), low density lipoprotein (LDL) and high density lipoprotein (HDL). The populations of these classes are very heterogeneous and each of them consists of several subclasses with different physical parameters and chemical composition of the core and outer monolayer. ${ }^{2,3}$ Dominant constituents in LDL core are cholesterol esters while in VLDL core are triglycerides. Common classification used in clinical practice recognizes two main plasma phenotypes found in general population, based on LDL characteristics. It was found that about $65-75 \%$ of population have the plasma of phenotype A, characterized by predominance of large
LDL particles, while in $20-30 \%$ of population plasma is of phenotype $\mathrm{B}$, with predominance of small dense LDL particles. ${ }^{4}$ It is known that small dense LDL particles are abundant with triglycerides which partially replace cholesterol esters in the core. ${ }^{3,5}$ Small LDL particles have decreased level of free cholesterol and phospholipids in the monolayer. While lipid and protein composition of LDL subclasses was extensively studied, the composition of VLDL and HDL particles present in phenotype B is still poorly known. There is a suggestion that small dense LDL particles are formed in several steps from large VLDL particles as precursors. ${ }^{3}$ These large particles, named $\mathrm{VLDL}_{1}$ in literature have about two times higher level of triglycerides and about three times lower level of cholesterol esters in their core, comparing with smaller $\mathrm{VLDL}_{2}$ particles which are precursors of large LDL particles. ${ }^{2} \mathrm{VLDL}_{1}$ particles have also reduced concentration of protein and phospholipids in the monolayer. According to this information, we expect that the dominant VLDL population in phenotype $\mathrm{B}$ belongs to $\mathrm{VLDL}_{1}$ particles. There is a common agreement that the people with phenotype B are at higher risk for the development of atherosclerosis, diabetes and cardiovascular disease. ${ }^{5,6}$ These processes

\footnotetext{
* Author to whom correspondence should be addressed. (E-mail: krilov@mef.hr)
} 
are based mainly on the influence of small atherogenic LDL particles.

In our earlier paper, we presented FT-IR spectroscopic studies of three lipoprotein classes and connected spectral characterization of each class with details of particle's structure. ${ }^{7}$ The attribution of lipid and protein bands in spectra was the basis for the spectral analysis in further studies.

The aim of this work was to look for the structural differences between VLDL, LDL and HDL lipoproteins, isolated separately from plasma of phenotype A and plasma of phenotype B. The details of the structural changes were observed in FT-IR spectra from particles obtained from two phenotypes. The results were compared with the structural data about lipoprotein subclasses from literature.

The differences in dynamics of oxidation for two types of particles were studied by ESR measuring the oxygen consumption.

\section{EXPERIMENTAL}

\section{Characterization of Plasma Phenotypes}

Plasma samples from individual patients were obtained from the Laboratory for Neurobiochemistry, Department for Special and Molecular Biochemistry at University Hospital Centre Zagreb. For the separation of LDL particle subclasses, electrophoretic method on horizontal, no denaturing, gradient $(3.5-5 \%)$ polyacrylamide gel has been developed. ${ }^{8}$ The method enables LDL phenotype determination in individual samples. For preparing a gradient gel, two types of crosslinking agents were combined: $N, N^{\prime}$-diallyltartardiamide (DATD) and N,N'-methylenebisacrylamide (BIS). Two different stock solutions, stock solution 1 with DATD: $T=20 \%, C=15 \%$, and stock solution 2 with BIS: $T=20 \%, C=4 \%$, were used for this preparation where $T$ is total monomer concentration defined as: $100 \times(m$ (acrylamide + crosslinker $) / g) /\left(\mathrm{V}_{\text {total }} / \mathrm{ml}\right)$ and $C$ is mass fraction of crosslinker defined as: $m$ (crosslinker) $/ m$ (acrylamide + crosslinker). Assembling cassette with Gel Bond PAG film placed between two glass plates was continuously filled with two working

Table 1. Volumes of WS for the preparation of gradient polyacrylamide gel sizes $225 \times 110 \mathrm{~mm}, 1 \mathrm{~mm}$ thickness

\begin{tabular}{lccc}
\hline & Stacking gel & Container 1 & Container 2 \\
\hline WS1 $^{(a)}(3.5 \%)$ & $8 \mathrm{ml}$ & $14 \mathrm{ml}$ & - \\
WS2 $(5 \%)$ & - & - & $14 \mathrm{ml}$ \\
TEMED & $10 \mu \mathrm{l}$ & $15 \mu \mathrm{l}$ & $15 \mu \mathrm{l}$ \\
APS $(40 \%)$ & $16 \mu \mathrm{l}$ & $15 \mu \mathrm{l}$ & $15 \mu \mathrm{l}$ \\
\hline
\end{tabular}

(a) WS1 contains $2.6 \mathrm{ml}$ of glycerol $(w=60 \%)$ for the formation of gradient gel.

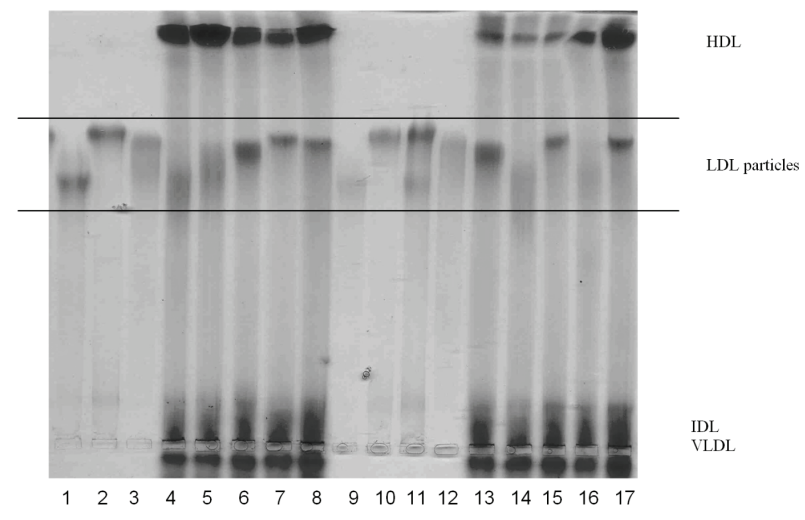

Figure 1. Separation of LDL particle subpopulations by horizontal gradient polyacrylamide gel electrophoresis $(3.5-5 \%)$. 1 and 9: LDL Standard H; 2, 10: LDL Standard A; 3, 12: LDL Standard B; $4-8,11$, and $13-17$ : plasma samples.

solutions (WS) of gel: WS1 with $3.5 \%$ gel solution and WS2 with $5 \%$ gel solution, Table 1.

Tetramethylethylenediamine (TEMED) was used with ammonium persulphate (APS) to catalyze the polymerization of acryl amide. APS was added immediately before gel preparation. After polymerization, the gel was used for electrophoresis.

Seven $\mu$ aliquots of plasma samples were applied on gel and low voltage electrophoresis (up to $175 \mathrm{~V}$ ) with $0.8 \mathrm{M}$ TRIS-borate buffer, $\mathrm{pH}=8.3$, was performed for about 18-20 hours. Each run was standardized by three LDL standards isolated by ultracentrifugation in density gradient: standard $\mathrm{H}$, whole range density $1.025-1.060 \mathrm{~g} / \mathrm{ml}$, standard A, density $1.025-1.044$ $\mathrm{g} / \mathrm{ml}$, and standard B, density $1.044-1.063 \mathrm{~g} / \mathrm{ml}$. Gels were stained with Coomassie blue R-250 and stored in distilled water up to evaluation. Evaluation of LDL subclasses was made by visual inspection of gels and densitometric scans with LKB 2202 Ultra Scan Laser Densitometer, presented in Figure 1.

\section{Lipoprotein Isolation}

Plasma of individual patients belonging to phenotype A or B was collected and pooled separately. Lipoproteins were isolated by the sequential ultracentrifugation. ${ }^{9}$ EDTA $(\gamma=1 \mathrm{~g} / 1)$ was added in the solution to protect lipoprotein against peroxidation. The cut-off density, $1.020 \mathrm{~g} \mathrm{~cm}^{-3}$ for VLDL, $1.063 \mathrm{~g} \mathrm{~cm}^{-3}$ for LDL, $1.21 \mathrm{~g}$ $\mathrm{cm}^{-3}$ for HDL was adjusted by adding potassium bromide. Each centrifugation procedure was performed at $50000 \mathrm{rpm}$ for 48 hours at $10^{\circ} \mathrm{C}$ in a Beckman $70 \mathrm{Ti}$ rotor. Before spectroscopic measurements, the lipoprotein solutions were dialyzed against degassed $0.01 \mathrm{M}$ phosphate buffer, $\mathrm{pH}=7.4$ and stored at $4^{\circ} \mathrm{C}$ in sealed syringes. The mass concentrations of solutions, in $\mu \mathrm{g}$ of protein per $\mu \mathrm{L}$ : 8.1 for VLDL-A, 1.55 for VLDL-B, 5.86 for LDL-A, 13.64 for LDL-B, 83.3 for HDL-A and 
87.2 for HDL-B were determined by the standard Lowry method. Before measurements, two lipoprotein solutions within each class were adjusted to equal concentration.

\section{FT-IR Spectroscopy}

The concentration of lipoprotein solution was rather low in order to avoid formation of particles' aggregates at higher concentration. For spectroscopic measurements, the lipoprotein solution $(2 \mu \mathrm{l})$ was placed on $\mathrm{ZnSe}$ window and dried under nitrogen for 15 minutes to obtain thin film. The film was further dried under vacuum for another 15 minutes. FT-IR spectra in transmission mode were recorded with the PerkinElmer Spectrum GX spectrometer equipped with a DTGS detector with 25 coadded scans in the region $4000-600 \mathrm{~cm}^{-1}$, with $4 \mathrm{~cm}^{-1}$ resolution, at room temperature. The spectrometer was continuously purged with nitrogen to remove the water vapor from the detector and sample compartment. The effect of drying the samples was checked by recording of spectra after different drying intervals. The spectra didn't change with prolonged drying procedure.

\section{ESR}

Concentration of oxygen in the lipoprotein solution was measured using 2,2,5,5-tetramethyl-3-pyroline-1-yloxy (CTPO) spin probe, obtained from Sigma-Aldrich. ${ }^{10}$ The resolution of super hyperfine coupling pattern from protons in methyl groups depends inversely on oxygen concentration. For oxidation measurements, $10 \mu \mathrm{l}$ of freshly prepared $\mathrm{CuCl}_{2}$ solution was added to $30 \mu \mathrm{l}$ of lipoprotein solution. The concentration of $\mathrm{CuCl}_{2}$ solution was adjusted to obtain the ratio of lipoprotein particles to copper ions 10:1. Low concentration of copper ions favors slow oxidation, which better mimics the lipoprotein medium in vivo. To each lipoprotein solution $40 \mu \mathrm{l}$ of CTPO solution was added, with final concentration $0.114 \mathrm{mmol} \mathrm{dm}^{-3}$ CTPO in the sample. The solutions were inserted into quartz capillaries which were sealed after that. In that way the amount of oxygen in capillaries was limited. ESR measurements were done at room temperature with a Bruker X-band ELEXYS 500 ESR spectrometer at room temperature. The oxygen concentration was determined from $K$ parameter of ESR spectra, using calibration curves. ${ }^{11}$

\section{RESULTS}

\section{FT-IR Spectroscopy}

FT-IR spectra of HDL particles isolated from plasma of phenotype A and phenotype B did not show any observable difference indicating that there are no observable

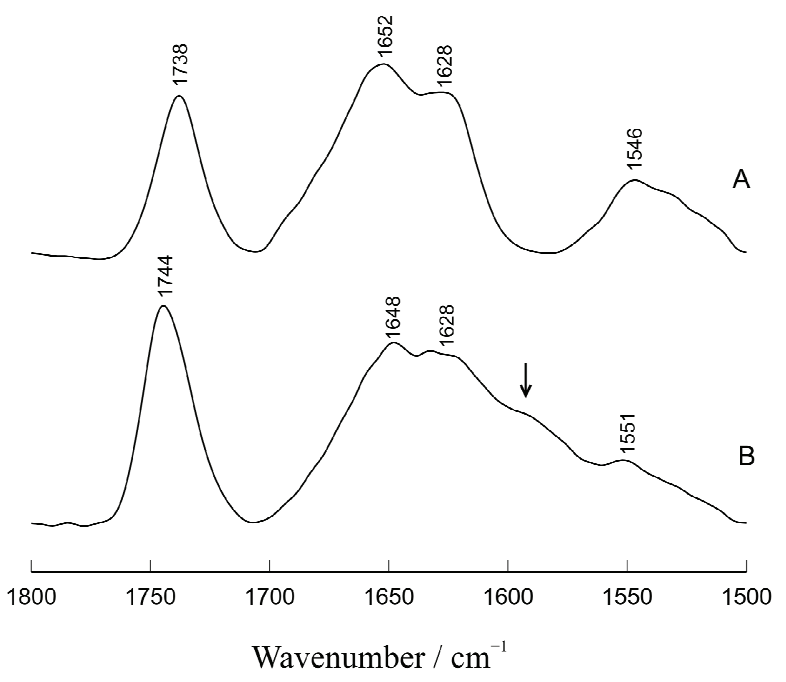

Figure 2. FT-IR spectra of LDL in solid film from phenotype A and phenotype $\mathrm{B}$, in $1800-1500 \mathrm{~cm}^{-1}$ region.

changes in the conformation and structure between these particles from two phenotypes.

However, in spectra of VLDL and LDL particles isolated from plasma of two phenotypes the differences were observed in lipid and in protein bands. FT-IR spectra of LDL in the region $1800-1500 \mathrm{~cm}^{-1}$ are presented in Figure 2.

The upper spectrum is from sample of phenotype A (LDL-A) and lower spectrum is from sample of phenotype $\mathrm{B}$ (LDL-B). The prominent band from lipid esters, $v(\mathrm{C}=\mathrm{O})$, is shifted from $1738 \mathrm{~cm}^{-1}$ in spectrum $\mathrm{A}$ to $1744 \mathrm{~cm}^{-1}$ in spectrum $\mathrm{B}$. The shift reflects the increase of ester vibrations from triglycerides in the core and decrease of vibrations from cholesterol esters and phospholipids in LDL-B. The difference in position of this band in LDL and VLDL was observed earlier. ${ }^{7,12}$ In addition, the relative intensity of this band compared with amide I band is higher in LDL-B reflecting the increased contribution of core lipids' vibrations to IR spectrum.

The profile of apo B-100 band is changed in B spectrum. Two characteristic peaks of amide I band are much less resolved due to the shift of left peak, from $1652 \mathrm{~cm}^{-1}$ in the spectrum A, to $1648 \mathrm{~cm}^{-1}$ in spectrum B. Amide II band at $1546 \mathrm{~cm}^{-1}$ in spectrum A is shifted to $1551 \mathrm{~cm}^{-1}$ in spectrum $\mathrm{B}$, with lower relative intensity. The broad shoulder at the right side of amide I band in spectrum B, indicated by arrow in Figure 2, around $1580 \mathrm{~cm}^{-1}$, comes from additional bands. It is attributed to vibrations from amino acid residues. Although $\mathrm{C}=\mathrm{C}$ stretching band from lipid chains is also present in this region, in liposome spectra it appeared as a triplet at $1645 \mathrm{~cm}^{-1}, 1595 \mathrm{~cm}^{-1}$ and $1548 \mathrm{~cm}^{-1}$ which was not observed here. ${ }^{7}$

Alterations in protein bands reflect the conformational changes in apo B-100 in LDL-B particles. 

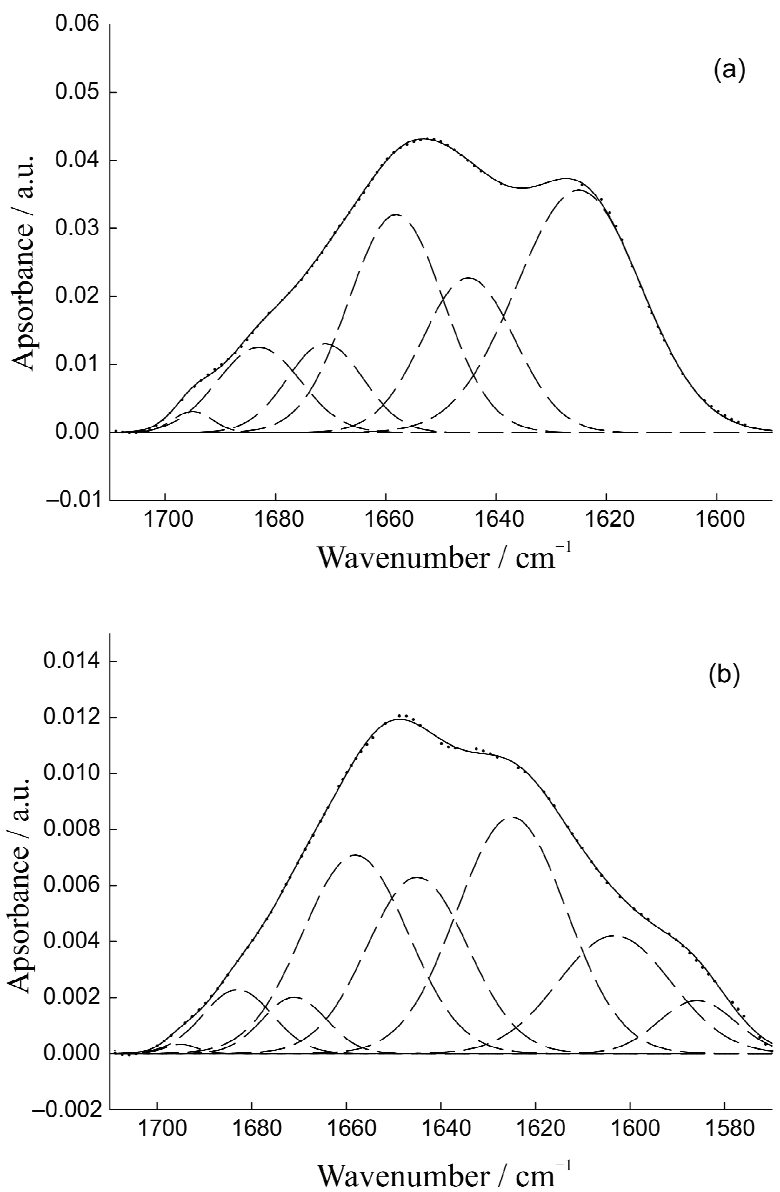

Figure 3. Decomposition of amide I band, solid curve, of apo B-100 from LDL-A (a) and LDL-B (b) into six component bands, dashed curves. Calculated amide I band is presented by dotted curve. The positions and ratios of component bands are listed in Table 2.

In order to better examine the structure of amide I band, the decomposition of the band into six component bands, Figure 3, was performed in the same way as earlier $^{7}$, for LDL-A, upper panel and LDL-B, lower panel. Table 2.

Parameters of component bands are presented in

Table 2. Decomposition of amide I band of apo B-100 from two LDL phenotypes

\begin{tabular}{cccccc}
\hline LDL-A & wavenumber $/ \mathrm{cm}^{-1}$ & $\%$ & LDL-B & wavenumber $/ \mathrm{cm}^{-1}$ & $\%$ \\
\hline$\alpha$-helix & 1658 & 26 & $\alpha$-helix & 1658 & 29 \\
$\beta$-sheet & 1695,1625 & 39 & $\beta$-sheet & 1695,1625 & 36 \\
$\beta$-turn & 1683,1671 & 17 & $\beta$-turn & 1683,1671 & 11 \\
random coil & 1645 & 18 & random coil & 1645 & 24 \\
& & amino acids & 1603 & \\
& & amino acids & 1585 & \\
\hline
\end{tabular}

Figure 4. FT-IR spectra of LDL in solid film from phenotype A, spectrum A, and phenotype B, spectrum B, in the 1300-600 $\mathrm{cm}^{-1}$ region.

The assignations were the same as in previous analysis of amide I band. ${ }^{7}$ The data for LDL-A are similar to those in earlier paper while the bands' positions and contributions for LDL-B are somewhat different. The largest deviation is the increase of random coil pattern and the decrease of $\beta$-turns. Two additional component bands were needed in order to simulate the slope of broad shoulder of amide I at lower frequency. One of these component bands is at $1585 \mathrm{~cm}^{-1}$ which corresponds to $v\left(\mathrm{COO}^{-}\right)_{\text {as }}$ from Asp residues. ${ }^{13}$

The differences in spectra from two LDL types were also observed in low frequency region, 1300-800 $\mathrm{cm}^{-1}$, Figure 4.

In this region the dominant bands are from phospholipids in monolayer: stretching vibrations of phosphate and choline group, and skeletal vibrations in their vicinity. ${ }^{14}$ The positions of both phosphate bands, $v\left(\mathrm{PO}_{2}^{-}\right)$, were changed from $1239 \mathrm{~cm}^{-1}$ and $1087 \mathrm{~cm}^{-1}$ in spectrum $\mathrm{A}$, to $1237 \mathrm{~cm}^{-1}$ and $1090 \mathrm{~cm}^{-1}$ in spectrum B. In addition, two bands from choline group, $v\left[\mathrm{C}-\mathrm{N}^{+}\left(\mathrm{CH}_{3}\right)_{3}\right]$, are shifted from $971 \mathrm{~cm}^{-1}$ and $840 \mathrm{~cm}^{-1}$

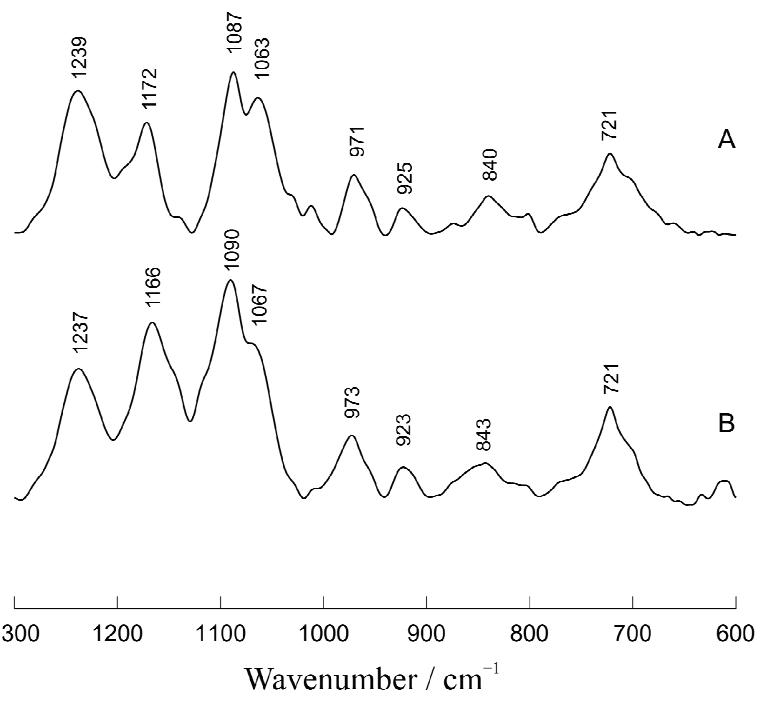



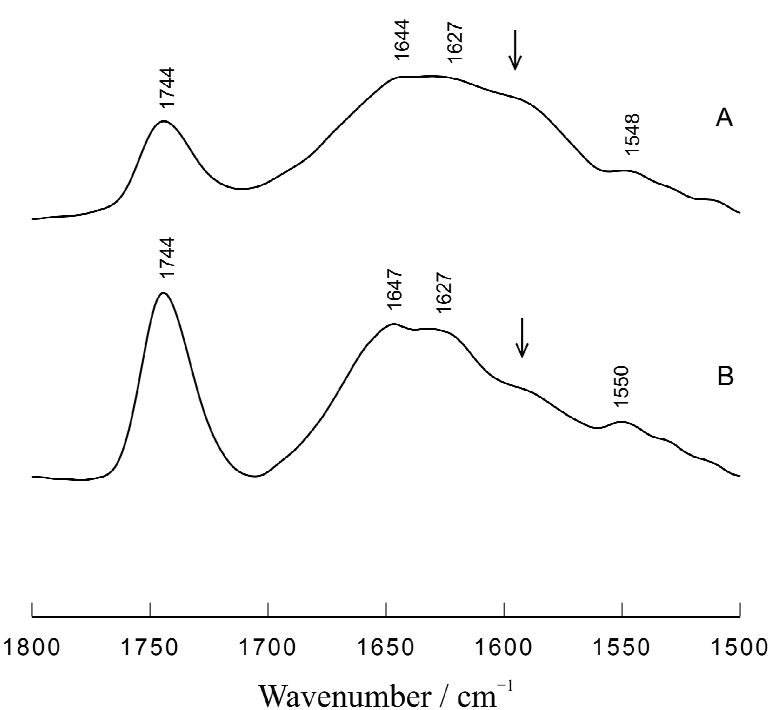

Figure 5. FT-IR spectra of VLDL in solid film from phenotype A, spectrum A, and phenotype B, spectrum B, in the 1800-1500 $\mathrm{cm}^{-1}$ region.

in spectrum A, to $973 \mathrm{~cm}^{-1}$ and $843 \mathrm{~cm}^{-1}$ in spectrum B. The positions of bands from skeletal vibrations in phospholipids' head part are also changed: $v(\mathrm{CO}-\mathrm{O})$ band was shifted from $1172 \mathrm{~cm}^{-1}$ in spectrum $A$ to $1166 \mathrm{~cm}^{-1}$ in spectrum $\mathrm{B}$. The positions of $\mathrm{C}-\mathrm{O}-\mathrm{C}$ stretching band were also different: at $1063 \mathrm{~cm}^{-1}$ in spectrum $\mathrm{A}$, and at $1067 \mathrm{~cm}^{-1}$ in spectrum B. The observed differences indicate that the polar part of phospholipids is sensitive to the conformational changes.

The profile of rocking doublet band from chain's $\mathrm{CH}_{2}$ groups at $721 \mathrm{~cm}^{-1}$ is slightly different in two spectra and relatively more pronounced and narrower in spectrum B.

Spectral differences between VLDL particles from two phenotypes are also readily observable. In the region $1800-1500 \mathrm{~cm}^{-1}$, Figure 5, the relative intensity of $\mathrm{C}=\mathrm{O}$ ester stretching band, in comparison with amide I band, is significantly higher in VLDL-B. This reflects the increased content of triglycerides VLDL-B. ${ }^{2}$

The profiles of amide I band are also different in two spectra. Amide I band in spectrum B is the same as spectrum B in Figure 2, but these bands are significantly different in spectrum $A$ in Figure 2 and spectrum $A$ in Figure 5. The higher number of vibrations from amino acid residues, indicated by arrow in Figure 5, could be responsible for the shape of amide I band in spectrum A. Amide II band is shifted from $1548 \mathrm{~cm}^{-1}$ in spectrum A to $1550 \mathrm{~cm}^{-1}$ in spectrum B. It is overlapped with amide I pattern in both spectra.

The differences between spectra from two phenotypes were also observed in the region $1300-600 \mathrm{~cm}^{-1}$, Figure 6.

The bands from the vibrations in lipid domain of VLDL particle are mostly more intensive in spectrum B.

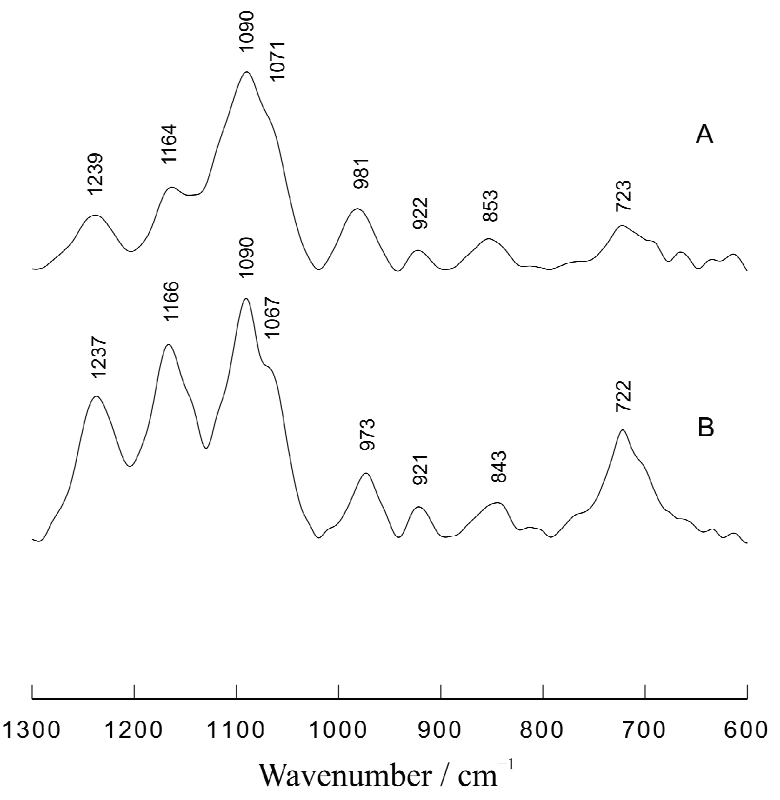

Figure 6. FT-IR spectra of VLDL in solid film from phenotype A, spectrum A, and phenotype B, spectrum B, in the 1300-600 $\mathrm{cm}^{-1}$ region.

Two bands from phosphate group are at $1239 \mathrm{~cm}^{-1}$ and $1090 \mathrm{~cm}^{-1}$ in spectrum $\mathrm{A}$, and at $1237 \mathrm{~cm}^{-1}$ and 1090 $\mathrm{cm}^{-1}$ in spectrum $\mathrm{B}$. The intensity ratio of antisymmetric and symmetric band is higher in spectrum B. The differences in positions of two bands from choline group are significant: at $981 \mathrm{~cm}^{-1}$ and $853 \mathrm{~cm}^{-1}$ in spectrum $\mathrm{A}$, and at $973 \mathrm{~cm}^{-1}$ and $843 \mathrm{~cm}^{-1}$ in spectrum $\mathrm{B}$. The $v(\mathrm{CO}-\mathrm{O})$ band from phospholipids' head was shifted from $1164 \mathrm{~cm}^{-1}$ in spectrum $\mathrm{A}$, to $1166 \mathrm{~cm}^{-1}$ in spectrum $\mathrm{B}$, and its intensity was increased. The $\mathrm{C}-\mathrm{O}-\mathrm{C}$ stretching band at $1071 \mathrm{~cm}^{-1}$ in spectrum A was shifted to $1067 \mathrm{~cm}^{-1}$ in spectrum $\mathrm{B}$. The band from rocking vibrations of $\mathrm{CH}_{2}$ groups at $723 \mathrm{~cm}^{-1}$ and $722 \mathrm{~cm}^{-1}$ is much stronger in spectrum $B$.

\section{ESR-Oxidation}

Oxygen concentrations, calculated from ESR spectral parameters of CTPO nitroxide radicals as a function of time are presented in Figure 7.

The time scale of oxidation process reflects the very slow or mild oxidation of lipoproteins. The data for HDL are presented in upper panel, for LDL in middle panel and for VLDL in bottom panel, for phenotype A as circles and for phenotype B as squares. Oxidation of LDL is much faster than other two lipoproteins and oxygen was almost completely spent in 8 days. There is practically no difference in the rate of oxidation between LDL-A and LDL-B. However, in HDL and VLDL, the oxidation was faster in samples from phenotype B. Moreover, the oxidation of VLDL was much slower than of HDL. In time course of 15 days, oxygen 


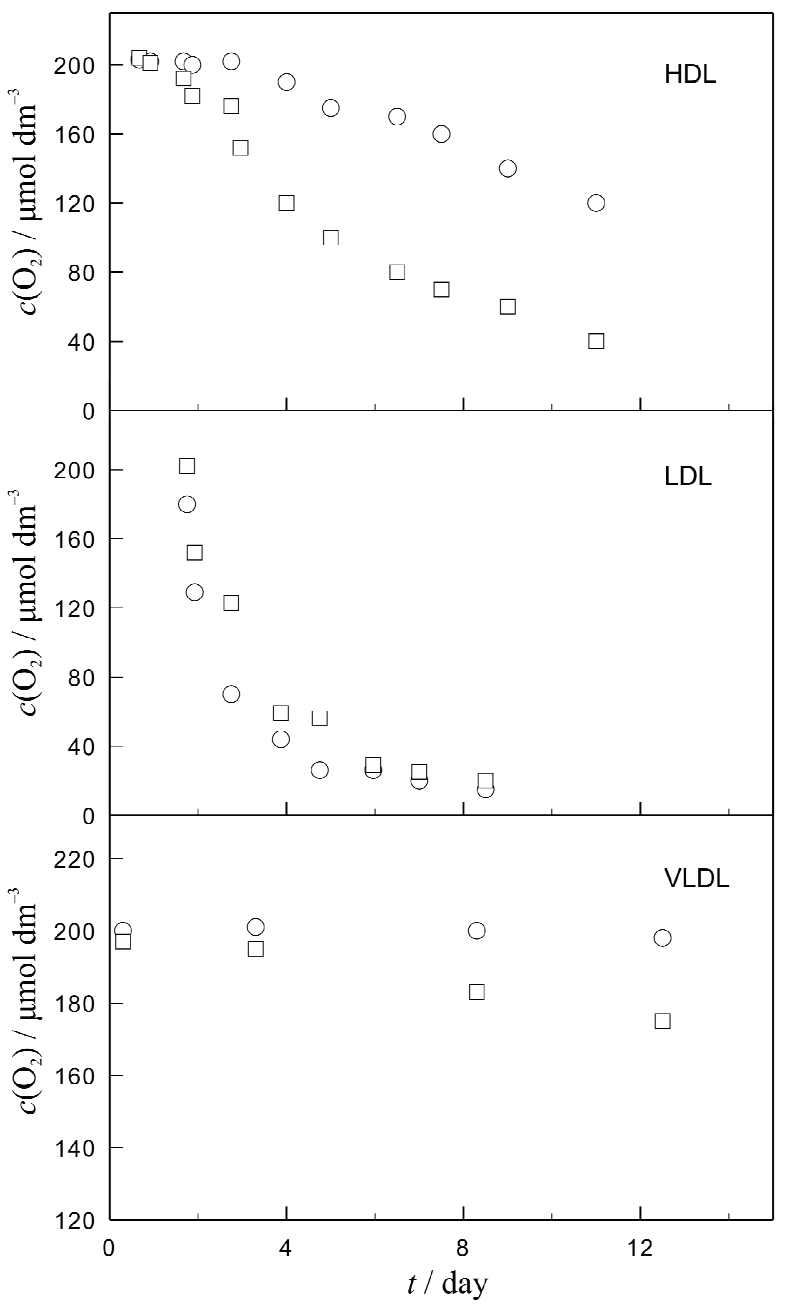

Figure 7. Oxygen consumption for HDL, LDL and VLDL solutions. Data presented in each panel are for solutions from phenotype A (०) and phenotype B ( $\square$ ).

was not consumed in VLDL-A and only about $23 \%$ in VLDL-B. In the same time interval, the concentration of oxygen dropped about $60 \%$ in HDL-A and $90 \%$ in HDL-B.

\section{DISCUSSION}

The knowledge about the composition of lipoprotein subclasses was mostly based on the information obtained from the concentration measurements of their components in plasma. Intention of our study was to investigate conformational differences between lipoproteins from phenotype A and B at the level of individual particle, using FT-IR spectroscopy.

Two main subclasses of HDL particles are $\mathrm{HDL}_{2}$, larger particles, and $\mathrm{HDL}_{3}$, smaller particles, although in some clinical studies a more detailed particle size distribution was obtained. ${ }^{4}$ It was found that the presence of large LDL particles was correlated with large HDL particles. The level of smaller HDL particles and smaller LDL particles was increased in plasma of patients with insulin resistance syndrome and type 2 diabetes. ${ }^{6}$ Changes occur mainly in protein domain of the particle.

In IR spectra of HDL particles from two phenotypes no differences were observed. It is known that two main apolipoproteins in HDL are apo A-I and apo A-II. In some pathological states chlorine, nitrogen and oxygen could be bound to protein changing its function. However, such additions do not change the protein conformation. The differences between mean HDL size for large particles $(8.99 \mathrm{~nm})$ and small particles $(8.42$ $\mathrm{nm})$ are too small to account for the changes in lipid ordering in particle's monolayer or the protein conformation.

For LDL and VLDL particles, differences in size and core and surface composition between subclasses require significant conformational changes of apo B-100 to preserve the stability of the particle. ${ }^{2}$ Moreover, the lower number of phospholipids and free cholesterol in LDL and VLDL from phenotype B induces changes in the head orientation and chain packing. This was reflected in IR spectra of LDL-A and LDL-B which showed apparent differences in the region 1800-800 $\mathrm{cm}^{-1}$, both in the bands of lipid and protein domain of the particle. The changes in protein domain of LDL-B are reflected in the position and profile of amide I and amide II bands and the increase in intensity of bands from amino acid residues. The surface area covered by lipids is about $36 \%$ smaller and therefore the area covered by protein is $49 \%$ larger than in large LDL particles. This enlargement forces apo B-100 to change its tertiary conformation in order to retain surface pressure unchanged and particle stable. ${ }^{2}$ From the composition of subclasses, it was calculated that the surface fraction of the amphipathic $\beta$ sheets and $\alpha$ helices, which are in contact with lipids, increases with the decrease of particle's size. ${ }^{15}$ That means the reduced thickness of protein which allows increased exposure of amino acid residues to external medium. The shift of the stretching band of ester group is the result of increased contribution of vibrations from triglycerides in LDL-B core and diminished contribution of surface lipids. These changes can be explained by the increase of the volume per molecule in the core, on the one side, and the increase of the surface area per molecule in the monolayer, on the other side, due to leakage of phospholipids and free cholesterol. These alterations are reflected in differences in other lipid bands presented in section Results. Some of them can be attributed to lipid chain ordering and packing, mostly deformation bands from the groups in acyl chains. The others demonstrate the differences in polar region of phospholipids. Both can be explained by a rearrangement of monolayer. 
IR spectra of VLDL from phenotype A and phenotype B demonstrated also spectral differences in lipid and protein domain. Carbonyl stretching band from lipid esters is much stronger in VLDL-B, indicating the abundance of triglycerides in its core. Contrary to LDL spectra, the contribution of vibrations from amino acid residues is larger in VLDL-A than in VLDL-B. The number of phospholipids and free cholesterols is lower in $\mathrm{VLDL}_{1}$ while its curvature is smaller in comparison with $\mathrm{VLDL}_{2}{ }^{3}$ That requires the different conformational change of apo B-100 than in LDL, i.e. its accommodation from the smaller to the larger VLDL particle. The apolipoprotein content in VLDL is only $8 \%$ and even lower in $\mathrm{VLDL}_{1}$. Therefore, it is possible that the majority of amino acid residues in $\mathrm{VLDL}_{1}$ are in contact with lipids and the contribution of their vibrations is smaller. In addition, the decrease of the amino acid band could be the influence of two other apolipoproteins found in VLDL-B, apo C-III and apo E. Apo E was detected in delipidated lipoprotein fraction with density corresponding to $\mathrm{VLDL}_{1}$, in electrophoresis separation on SDS polyacrylamide gel, but not in other fractions. ${ }^{16}$

At lower frequencies, the increase of lipid bands from chains' deformation vibrations in VLDL-B can be attributed to much higher triglyceride content in the core. Additionally, bands from vibrations in polar head of phospholipids are stronger in VLDL-B samples due to conformational changes caused by decrease of curvature.

The rate of oxidation of LDL, measured here as oxygen consumption, was almost the same in LDL-A and LDL-B, although it was expected that small dense LDL particles, due to their atherogenic character, are more sensitive to oxidation. ${ }^{17}$ In addition it was found that the surface monolayer is more vulnerable to oxidation in dense LDL particles than in buoyant LDL-A particles. The stronger curvature of the surface of LDL$\mathrm{B}$ in comparison with LDL-A, could enable the lipid radicals in phospholipids domain of LDL-B to mutually interact with the formation of non radical products and therefore stopping the further oxygen consumption. The number of such events is lower in LDL-A and therefore oxygen consumption rate could be similar in both phenotypes. In HDL and VLDL the oxidation is obviously faster in particles from phenotype B. Oxidation of VLDL is much slower process than for other two lipoprotein classes. Moreover, the distinction between oxidation rate for VLDL-A and VLDL-B could be observed. In the other experiments in the conditions for strong oxidation of VLDL with copper ions, it was also found out that the oxidation rate was slower than for LDL particles, under same conditions. ${ }^{18}$ These results suggest that all three types of lipoproteins are involved in oxidation process which could be connected with the atherogenic alterations in patients with plasma of phenotype B.

\section{CONCLUSION}

Human plasma contains LDL particles of various size and composition. In clinical routine, plasma is usually classified in two phenotypes A, with distribution of larger LDL particles and B, with distribution of smaller LDL particles. In this paper we presented the results of structural analysis of all three major lipoproteins in plasma which were isolated separately from plasma phenotype A and plasma phenotype B. Structural differences in FT-IR spectra of LDL and VLDL were observed in the both, protein and lipid domains. That was confirmed by differences in their FT-IR spectra, which are explained by the change of relative contribution of particular lipid and protein components which required the conformational rearrangement in monolayer. Due to intertwined metabolic pathways and mutual exchange of protein and lipid molecules, some structural differences in HDL were also expected, but were not observed in IR spectra. The study of oxidation dynamics of these three lipoproteins, demonstrated the higher sensitivity to oxidation for particles from phenotype B with exception of LDL where oxidation was very fast in particles from both phenotypes.

Acknowledgements. This work was supported by Ministry of Science, Education and Sports of the Republic of Croatia (project No. 108-1080134-3105). We acknowledge the Laboratory for Neurobiochemistry, Department for Special and Molecular Biochemistry at Clinical Hospital Centre Zagreb for the donation of selected plasma samples from individual patients. Additionally, we acknowledge Laboratory for Second Messengers, Department for Chemistry and Molecular Biology, Croatian Institute for Brain Research, for the use of ultracentrifuge for the lipoprotein isolation, Department of Biophysics, Faculty of Pharmacy and Biochemistry, for the use of ESR spectrometer and Department for Chemistry and Biochemistry University of Zagreb School of Medicine for the performing concentration measurements of lipoproteins.

\section{REFERENCES}

1. M. H. Dominiczak, Apolipoproteins and Lipoproteins in Human Plasma, in: N. Rifai and G. R. Warnick (Eds.), Laboratory Measurement of Lipids, Lipoproteins and Apolipoproteins, AACC Press, Washington DC (1994) 1-20.

2. J. R. McNamara, D. M. Small, Z. Li, and E. J. Schaefer, J. Lipid Res. 37 (1996) 1924-1935.

3. K. K. Berneis and R. M. Krauss, J. Lipid Res. 43 (2002) 1363-1379.

4. K. R. Feingold, C. Grunfeld, M. Pang, W. Doerrler, and R. M. Krauss, Arterioscler. Thromb. Vasc. Biol. 12 (1992) 1496-1502.

5. S. Dejager, E. Bruckert, and M. J. Chapman, J. Lipid Res. 34 (1993) 295-308.

6. W. T. Garvey, S. Kwon, D. Zheng, S. Shaughnessy, P. Wallace, A. Hutto, K. Pugh, A. J. Jenkins, R. L. Klein, and Y. Liao, Diabetes 52 (2003) 453-462.

7. D. Krilov, M. Balarin, M. Kosović, O. Gamulin, and J. BrnjasKraljević, Spectrochim. Acta, Part A 73 (2009) 701-706. 
8. M. Trbojević-Čepe, Ž. Vogrinc, D. Horvat, and A. Stavljenić, CCLM, Special Supplement - abstracts of EUROMEDLAB 2001 39 (2001), S 292.

9. J. Brnjas-Kraljević, G. Pifat, and J. N. Herak, Croat. Chem. Acta 66 (1993) 547-554.

10. W. K. Subczynski and J. S. Hyde, Biophys. J. 45 (1984) 743-748.

11. C. S. Lai, L. E. Hopwood, J. S. Hyde. S. Lukiewicz, Proc. Natl. Acad. Sci. U.S.A. 79 (1982) 1160-1170.

12. M. Nara, M. Okazaki, and H. Kagi, Chem. Phys. Lipids 117 (2002) 1-6.
13. A. Barth, Prog. Biophys. Mol. Biol. 74 (2000) 141-173.

14. W. Hübner and H. H. Mantsch, Biophys. J. 59 (1991) 1261-1272.

15. J. P. Segrest, M. K. Jones, H. De Loof, and N. Dashti, J. Lipid Res. 42 (2001) 1346-1367.

16. T. A. Musliner, C. Giotas, and R. M. Krauss, Arterioscler. Thromb. Vasc. Biol. 6 (1986) 79-87.

17. D. L. Tribble, R. M. Krauss, M. G. Lansberg, P. M. Thiel, J. J. M. van den Berg, J. Lipid Res. 36 (1995) 662-671.

18. N. Dousset, M. Taus, G. Ferretti, J. C. Dousset, G. Curatola, P. Valdiguié, Biochem. Mol. Biol. Int. 45 (1998) 1021-1030. 